\title{
Rotavirus VP4 Genotypes and Phylogenetic Analysis Among Strains Recovered From Children, Admitted With Acute Gastroenteritis in Bahrami Children's Hospital, Tehran, Iran
}

\author{
Aliakbar Rahbarimanesh ${ }^{1,}$; Hassanali Saberi ${ }^{1}$; Sharzah Modarres ${ }^{2}$; Payman Salamati ${ }^{1}$; \\ Hoshang Akhtar-Khavari ${ }^{1}$; Zahra Haghshenas ${ }^{1}$; Behdad Navabi ${ }^{1}$ \\ ${ }_{2}^{1}$ Department of Pediatrics, Bahrami Children's Hospital, Tehran University of Medical Sciences, Tehran, IR Iran \\ ${ }^{2}$ Department of Virology, Pasteur Institute, Tehran, IR Iran \\ *Corresponding author: Aliakbar Rahbarimanesh, Department of Pediatrics, Bahrami Children's Hospital, Tehran University of Medical Sciences, Tehran, IR Iran. Tel:+98-2177568809, \\ Fax:+98-2177568809, E-mail: rahbarim@tums.ac.ir
}

Received: December 4, 2013; Accepted: December 26, 2013

\begin{abstract}
Background: Rotavirus infection is one of the most common causes of severe dehydrating diarrhea in children with a significant health burden on them, in Middle Eastern countries, including Iran.

Objectives: We aimed to investigate P serotype of group A rotavirus variability among children hospitalized with acute gastroenteritis (AGE), track the changes in the genotypes of circulating viruses, and elucidate the efficacy of introducing the commonly accepted rotavirus vaccines to the national vaccination program.

Patients and Methods: All children 14 years or younger, admitted in the Infectious Disease Ward of Bahrami Children's Hospital due to acute nonbloody gastroenteritis between January 2009 and January 2010, were included in this study. Stool samples were collected and specific rotavirus genotype primers were defined by Virology Lab of the Pasteur Institute. The specimens were assessed for rotavirus using RNA-PAGE and semi-nested multiplex molecular virology techniques.

Results: Twenty nine rotavirus strains were retrieved from 150 samples collected from hospitalized children with gastroenteritis (14 males and 15 females; mean age $20.9 \pm 18.19$ months) were included in the study. The most frequent P serotype was P8 (86.2\%), followed by P6 (6.9\%) and P4 (6.9\%). In addition, the majority of strains (89.65\%) exhibited a long electrophore-type pattern on the PAGE system. Rotavirus was mostly isolated from children aged less than 2 years (86.2\%), and during winter (44.7\%). No significant relationships were observed between the rotavirus infection and; age, sex, season, or initial signs and symptoms such as fever, estimated dehydration on admission, nausea, and vomiting.

Conclusions: Results of the current study supported the potential efficacy of introducing RotaTeq and Rotarix vaccines to the Iranian children vaccination program. We also propose prospective studies on confirmed rotavirus diarrheal illness in various parts of Iran, to determine the distribution of various genotypes across the country.
\end{abstract}

Keywords:Diarrhea; Gastroenteritis; Rotavirus; Children; Genotype

\section{Background}

Diarrheal illness remains one of the top five causes of death in lower and middle-income countries, especially in children younger than 5 years (1). Lack of routine clinical testing for these viruses limits our understanding of their roles (2). Furthermore, the rotavirus has been the leading cause of acute gastroenteritis (AGE) in children worldwide over the past two decades $(3,4)$. Based on national estimates of rotavirus attributable deaths among children $<5$ years, the cumulative global death toll was approximately 527000 in 2004 (5), and 453000 in 2008, ranging from 98621 (India) to fewer than 5 deaths (74 countries) (6).

Rotavirus, a member of the Reoviridae family, has a tri- ple-layered capsid enclosing an 11-segment double-strand RNA (dsRNA) genome, that encodes six viral and six nonstructural proteins $(7,8)$. It is differentiated into at least seven species, i.e. rotavirus A-G, and three are capable of affecting humans (groups A-C) $(9,10)$. Group A is the major pathogen of severe gastroenteritis in infants and young children $(7,9,10)$. Serological types of group A rotavirus are determined through its inner capsid protein (VP6) and outer capsid proteins (VP7, VP4). Based on antigenicity of the outer capsid proteins, namely VP7 and VP4, two independent serotype systems, i.e. $G$ and P, have been established $(9,10)$. So far, at least 31 different $P$ types, and 23 different $\mathrm{G}$ types have been reported (11).

Implication for health policy/practice/research/medical education:

These types of studies are important in order to motivate researchers to investigate the VP4 genotype variability of group A rotavirus strains among children hospitalized with acute gastroenteritis.

Copyright (C) 2014, Iranian Society of Pediatrics. This is an open-access article distributed under the terms of the Creative Commons Attribution License, which permits unrestricted use, distribution, and reproduction in any medium, provided the original work is properly cited. 
Various $\mathrm{G}$ and $\mathrm{P}$ type rotaviruses have originated from different animal species. In humans, rotaviruses G1, G2, $\mathrm{G} 3$ and $\mathrm{G} 4$ are the most prevalent $\mathrm{G}$ types reported worldwide $(9,10)$. Considering the prevalence, P4, P6, and P8, are the most commonly isolated P serotypes collected from humans $(7,9,10,12)$. Usually P4 is associated with G2, while P8 is linked with G1, G3, or G4, and P6 is found with any of the G1to G4 types (9). Generally, owing to typical varieties among virus families and even inside families, the development of broadly applicable vaccines is challenging (13). Epidemiologic surveillance of the $G$ and P serotypes to identify the most prevalent types is an important step in developing rotavirus vaccines (9).

The success of a rotavirus vaccination program depends on the development of robust regional surveillance systems that provide comprehensive and systematic data on the disease burden (12). To date, two vaccines are currently FDA-approved, namely Rotarix and RotaTeq (14). The two-dose Rotarix vaccine is composed of a single component, an attenuated human rotavirus with G1P8, which is the most prevalent type of human rotavirus; and RotaTeq which consists of five reassortant rotavirus strains and contains the major antigenic types of human rotavirus, i.e. G1-G4, and P8 (7, 9).

Although there have been some nationally based reports on the molecular epidemiology of rotavirus diarrhea in Iranian children, continuous surveillance studies are important not only as a means of monitoring new genotypes, but also to track changes in circulating genotypes. In addition, on the basis of data collected from 44 studies carried out in the Middle East and North Africa, rotavirus impose a heavy health burden on children less than five years-of-age (15), however, current burden of illness data specific to the Middle East and North Africa is limited (15).

\section{Objectives}

The aim of this study was to investigate VP4 genotype variability of group A rotavirus strains among children hospitalized with AGE in the Bahrami Children's Hospital, Tehran, Iran, from January 2000 to January 2010.

\section{Patients and Methods}

This study was conducted as a case-series to recruit all children from birth to 14 years-of-age admitted to the Infectious Disease Ward of Bahrami Children's Hospital, due to acute non-bloody gastroenteritis, in the time period January 2009 to January 2010. It should be mentioned that based on the hospital's protocols, acute diarrheal cases are admitted to the Infectious Disease Ward based on the following criteria: moderate to severe dehydration, and/or unable to tolerate oral hydration despite initial rehydration. Nevertheless, later on, children with nosocomial, chronic diarrhea, food intolerance, and diarrhea related to antibiotics, were excluded from the study.
In this study, diarrhea was defined as the passage of three or more loose or watery stools per day (16), though absolute limits of normalcy were difficult to define. The study lasted a full year to cover all of the seasons of a year, in order to oversee the seasonal variability of rotavirus diarrhea. To improve recording of the intended variables, and track the rotavirus infected cases, a questionnaire was designed and implemented. The questionnaire included demographic data: age, sex, date, and a summary of signs and symptoms at the time of admission; estimated level of dehydration, fever, and the presence of nausea and vomiting.

All of the patients and their parents were informed by the head physicians regarding the study and its nature, and consented to participate and share their anonymous data. Upon consenting, a stool sample was collected immediately, stored at less than $-4{ }^{\circ} \mathrm{C}$, and finally transferred to the Virology Lab of the Pasteur Institute to be investigated further. Specimens were assessed for rotavirus using RNA-PAGE, and semi-nested multiplex molecular virology techniques, in order to define specific rotavirus genotype primers. Following nucleotide sequencing, the phylogenetic analysis was graphed by means of GeneBank standards.

We used SPSS version 17 for statistical analysis and reporting of the results. Moreover, data of the children's ages was categorized into five distinct groups; birth to 6 months, 6 months to 1 year, 1 - 2 years, 2 - 5 years, and lastly 5 - 14 years. Categorical variables were described as; frequencies, proportions and continuous variables, and reported as; means, standard deviations, minimum and maximum. Bivariate analysis was used to compare rotavirus positive and rotavirus negative, patient characteristics were analyzed using Kolmogorov-Smirnov, MannWhitney $U$, and chi-square statistical tests. In this study, $P$ values of less than 0.05 were considered as statically significant.

All research ethics and regulations adopted by the Iranian Medical Commission of Scientific Research were considered in every step of this study and researchers adhered to these. Academic honesty and trustworthiness, impartiality and avoiding certain trends have been respected; however, the study was not posed with any other ethical restriction. The study did not require review by the Tehran University of Medical Sciences Review Board.

\section{Results}

In total, 150 stool specimens from children (57 girls and 93 boys) less than 14 years (1.5 months to 9 years; mean age of $20.9 \pm 18.19$ months), who were admitted to the Infectious Disease Ward of Bahrami Children's Hospital with a diagnosis of acute non-bloody gastroenteritis, from January 2009 to January 2010, were examined. Finally, rotavirus group A was found in 29 specimens (19.3\%) of the recruited cases. 
The majority of the rotavirus group A cases were isolated from children younger than two years-of-age (total of 25 case: $86.2 \%$ ), mostly in the 6 months to 24 months age range (Table 1) (total of 24 case: $83 \%$ ); nevertheless, rotavirus cases showed no statistically significant (P-value $=0.0928$ ) difference among the various age groups. On the basis of the affirmed cases, there was no statistical significant difference in gender (14 boys versus 15 girls; P-value $=0.21)$. From the seasonal view point, most cases (44.7\%) were collected in winter, followed by fall (26\%), and spring (16.7\%); however, this also exhibited no statistically significant difference.

Retrospective admission time signs and symptoms in the rotavirus group A acute gastroenteritis-confirmed cases included; fever in $82.7 \%$ (24 cases) (P value $=1$ ), nausea and vomiting in $89.6 \%$ (26 cases) ( $P$ value $=1$ ), and moderate dehydration in $86.2 \%$ (25 cases) (P-value $=1$ ), were the main estimated initial dehydration types.

Based on ds RNA genomic analysis, and 10 and 11 segment migration profile patterns on a PAGE system, the majority (89.65\%) of isolated strains (26 strains), showed a long electrophoresis type pattern, and less than 10\% (3) exhibited a short electrophore type pattern. Finally, all extracted strains were serotyped in the P system, and P8 was the dominant P serotype (86.2\%) (25 cases), thereafter P6 and P4 were both found in 6.9\% (2 cases) of collected strains (Table 2).

To compare the recovered sequences in our study with that of other countries, a phylogenetic tree (Figure 1) was constructed using the neighbor-joining method with 1000 bootstrap replicates implemented in Molecular Evolutionary Genetics Analysis (MEGA) software.

Table 1. Age Group Frequency in Rotavirus Acute Gastroenteritis, Nonrotavirus Acute Gastroenteritis, and Total Population

\begin{tabular}{llll}
\hline Age & Rotavirus AGE $^{\text {a }}$, Frequency $(\%)$ & Non- Rotavirus AGE, Frequency (\%) & Study Population, Frequency (\%) \\
\hline $\mathbf{0}$ - $\mathbf{6}$ mo & $1(3.4)$ & $15(12.4)$ & $16(10.7)$ \\
$\mathbf{6}$ - 12 mo & $10(34.5)$ & $40(33.1)$ & $50(33.3)$ \\
$\mathbf{1 - 2}$ y & $14(48.3)$ & $26(21.5)$ & $40(26.7)$ \\
$\mathbf{2}$-5 y & $4(13.8)$ & $34(28.1)$ & $38(25.3)$ \\
$\mathbf{5}$ - 14 y & $0(0)$ & $6(5.0)$ & $6(4.0)$ \\
Total & $29(100)$ & $121(100)$ & $150(100)$ \\
\hline
\end{tabular}

a Abbreviation: AGE, acute gastroenteritis.

Table 2. Rotavirus Sequencing and Their Distribution in Various Seasons

\begin{tabular}{llllll}
\hline & Spring, Frequency (\%) & Summer, Frequency (\%) & Fall, Frequency (\%) & Winter, Frequency (\%) & Total, Frequency (\%) \\
\hline P8 & $6(20.7 \%)$ & $3(10.3 \%)$ & $7(24.1 \%)$ & $9(31.0 \%)$ & $25(86.2 \%)$ \\
P4 & $0(0.0 \%)$ & $0(0.0 \%)$ & $0(0.0 \%)$ & $2(6.9 \%)$ & $2(6.9 \%)$ \\
P6 & $0(0.0 \%)$ & $0(0.0 \%)$ & $1(3.4 \%)$ & $1(3.4 \%)$ & $2(6.9 \%)$ \\
Total & $6(20.7 \%)$ & $3(10.3 \%)$ & $8(27.6 \%)$ & $12(41.4 \%)$ & $29(100.0 \%)$ \\
\hline
\end{tabular}

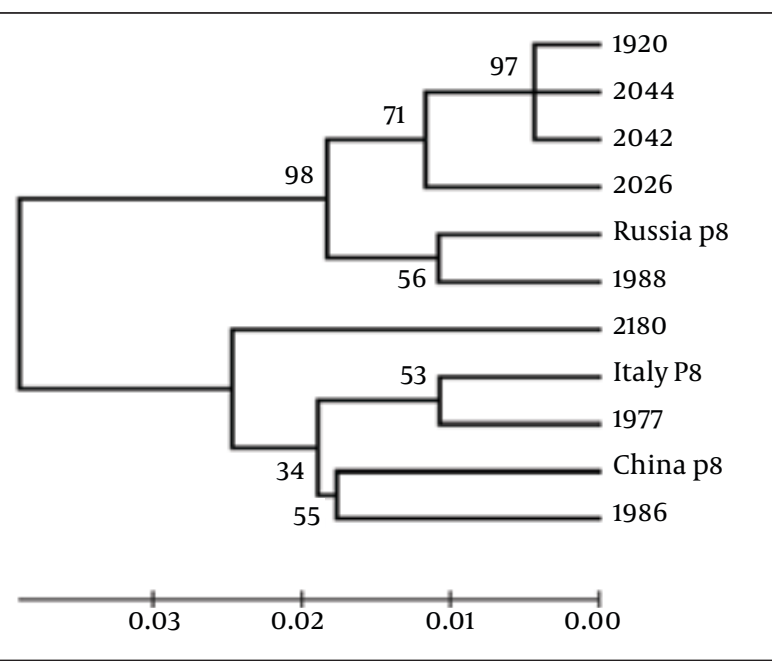

Figure 1. P8 Rotavirus Sequence Phylogenetic Tree Using the NeighborJoining Method
Upon phylogenetic analysis of the selected P8 rotavirus strains, we found that the retrieved sample from our study was most closely related to those reported by Asian and European countries, including; Russia, China, and Italy.

\section{Discussion}

Rotavirus infection remains one of the most common causes of severe dehydrating diarrhea worldwide among children, particularly in children under 5 years $(17,18)$ and it accounts for $20 \%-70 \%$ of diarrheal-associated hospitalizations $(19,20)$. Despite a less defined seasonal distribution variation, patterns of infection in temperate climates demonstrate peaks mostly in winter and lows in summer; on the other hand, it has a more consistent pattern in tropical environments (21). Based on the most recent WHO report (6), deaths due to rotavirus in proportion to all deaths in children younger than five, range from 13.8\% (in Afghanistan) to almost zero in 27 countries; Iran is ranked as 43rd with a rate of 5.3 deaths in 
children less than five years (6). The stated rate necessarily supports the need for further studies on this issue, and undoubtedly motivated the researchers of this study to investigate rotavirus and its circulating VP4 genotypes. Our study was conducted among a group of children admitted for dehydrating non-bloody diarrhea to Bahrami Children's Hospital, which serves children from regions mostly located in the east of Tehran.

Continuous surveillance of circulating rotavirus strains is definitely an essential step before and after introducing a vaccine in preventative national health system programs. This is important, primarily to identify the epidemiologically dominant rotavirus genotypes throughout the region $(22,23)$, to examine the efficacy of the vaccine $(24,25)$, and last but not least to track and record the spread of uncommon rotavirus strains (22), as found by Farahtaj et al. (26), which is in contrast to previous reports in Iran. All of the above are key contributing factors required to improve effective preventive measures (17). Rotavirus induced diarrhea, unlike the bacterial one, is prevalent in both temperate and tropical regions. It exhibits a distinct winter seasonality in temperate areas known as 'winter diarrhea,' primarily in the Americas, and regions with a variety of climates such as Toronto, Ontario, Canada and Houston, Texas, USA (27, 28); on the other hand, little is known regarding its seasonal variability in tropical areas $\left(24^{\circ} \mathrm{N}-24^{\circ} \mathrm{S}\right)(28,29)$; although a meta-analysis in the tropics suggests that the rotavirus infection rate tends to be highest under cool, dry conditions in these areas (29). Previous studies that have taken place in Iran $\left(27^{\circ} \mathrm{N}-38^{\circ} \mathrm{N}\right)$ reported a winter-seasonal-outbreak consistent with our study (Khalili et al. in Shahrekord (30), Hamedi et al. in Mashhad (31), and Ghorashi et al. in Tabriz (32)), or a spring-seasonal-outbreak (Amini et al. in Tehran (33), and Zarnani et al. in Tehran (34)). Our study observed that a seasonal drift might be due to a range of local climatological factors (29), which might have altered since 1990 (30), or 2003 (34), till our study in 2010; however, more research needs to be done in order to find conclusive reasons.

We found rotavirus in $19.3 \%$ of the study population, but the reported rates vary widely among the past national studies. Previously, the rotavirus infection rate has been shown to have a wide range: $78 \%$ by Khalili et al. in Shahrekord (30), 55.6\% by Ghorashiet et al. in Tabriz (32), $28.8 \%$ by Hamedi et al. in Mashhad (31), 26.3\% by Samarbafzadeh et al. in Ahvaz (35), 28.5\% by Momenzadeh et al. in Tehran (36), 25\% by Amini et al. in Tehran (33), 24.6\% by Farahtaj et al. in Tehran (26), 19\% by Modaress et al. in Tehran (12), and 15.3\% by Zarnani et al. in Tehran (34).

This wide spectrum of prevalence across the country stresses the need for continuous epidemiological studies. Moreover, research studies in Tehran have been representative of rotavirus frequency in a band of $15.3 \%-28.5 \%$ from 1998 (33), to our study in 2010.

The G1P8 genotype has been reported as the most preva- lent rotavirus genotype in many of the past international studies (18, 37-39). In addition, P8 has also been reported by many national studies, including Khalili et al. (74\% in 146 strains) (30), Modaress et al. (66.3\% in 133 strains) (12), and Farahtaj et al. (59.2\% G1P8 - SGII in 92 strains) (26), as the most prevalent observed rotavirus genotype in Iran. As stated previously, our results identified the P8 genotype as the most prevalent (86.2\%) collected strain.

We recommend prospective studies on confirmed cases of rotavirus diarrheal illness from various parts of Iran to identify the distribution of various genotypes across the country. We also suggest adding currently licensed RotaTeq and Rotarix vaccines containing G1P8 to the vaccination components, since the results of the current study along with previous studies in the region support the potential efficacy of introducing RotaTeq and Rotarix vaccines to the Iranian children vaccination program.

\section{Acknowledgements}

We thank the staff of Virology Laboratory of Pasteur Institute, and emergency department and clinic staff of Bahrami Children's Hospital, Tehran, Iran, that helped us in finding and referring eligible patients for stool sampling.

\section{Authors' Contribution}

All authors contributed equally in this study.

\section{Financial Disclosure}

Authors had no financial interests related to the material in this manuscript.

\section{Funding/Support}

Authors did not receive support from any organization.

\section{References}

1. Monroe SS. Control and prevention of viral gastroenteritis. Emerg Infect Dis. 2011;17(8):1347-8.

2. Hall AJ, Rosenthal M, Gregoricus N, Greene SA, Ferguson J, Henao $\mathrm{OL}$, et al. Incidence of acute gastroenteritis and role of norovirus, Georgia, USA, 2004-2005. Emerg Infect Dis. 2011;17(8):1381-8.

3. Parashar UD, Burton A, Lanata C, Boschi-Pinto C, Shibuya K, Steele D, et al. Global mortality associated with rotavirus disease among children in 2004. J Infect Dis. 2009;200 Suppl 1:S9-15.

4. Glass RI, Bresee JS, Turcios R, Fischer TK, Parashar UD, Steele AD. Rotavirus vaccines: targeting the developing world. J Infect Dis. 2005;192 Suppl 1:S160-6.

5. Who Publication. Rotavirus surveillance worldwide 2009. Weekly Epidemiological Record; 2011. 174-6.

6. Estimated rotavirus deaths for children under 5 years of age. 2008 Available from: http://www.who.int/immunization_monitoring| burden/rotavirus_estimates/en/.

7. Shim JO, Baek IH, Le VP, Ko EM, Seok WS, Uh Y, et al. Molecular characterization of rotavirus diarrhea among children in South Korea: detection of an unusual G11 strain. Arch Virol. 2011;156(5):887-92.

8. Nakagomi O, Nakagomi T, Akatani K, Ikegami N. Identification of rotavirus genogroups by RNA-RNA hybridization. Mol Cell Probes. 1989;3(3):251-61. 
9. Mori T, Nomori H, Ikeda K, Kobayashi H, Iwatani K, Yoshioka M, et al. Microscopic-sized "microthymoma" in patients with myasthenia gravis. Chest. 2007;131(3):847-9.

10. Ogilvie I, Khoury H, Goetghebeur MM, El Khoury AC, Giaquinto C. Burden of community-acquired and nosocomial rotavirus gastroenteritis in the pediatric population of Western Europe: a scoping review. BMC Infect Dis. 2012;12:62.

11. Han TH, Kim CH, Chung JY, Park SH, Hwang ES. Genetic characterization of rotavirus in children in South Korea from 2007 to 2009. Arch Virol. 2010;155(10):1663-73.

12. Modaress S, Rahbarimanesh AA, Edalat R, Sohrabi A, Modarres S, Gomari $\mathrm{H}$, et al. Human rotavirus genotypes detection among hospitalized children, a study in Tehran, Iran. Arch Iran Med. 2011;14(1):39-45

13. Ojosnegros S, Beerenwinkel N. Models of RNA virus evolution and their roles in vaccine design. Immunome Res. 2010;6 Suppl 2:S5.

14. Krishnarajah G, Davis EJ, Fan Y, Standaert BA, Buikema AR. Rotavirus vaccine series completion and adherence to vaccination schedules among infants in managed care in the United States. Vaccine. 2012;30(24):3717-22

15. Khoury H, Ogilvie I, El Khoury AC, Duan Y, Goetghebeur MM. Burden of rotavirus gastroenteritis in the Middle Eastern and North African pediatric population. BMC Infect Dis. 2011;11:9.

16. King CK, Glass R, Bresee JS, Duggan C, Centers for Disease C, Prevention. Managing acute gastroenteritis among children: oral rehydration, maintenance, and nutritional therapy. MMWR Recomm Rep. 2003;52(RR-16):1-6.

17. Nakawesi JS, Wobudeya E, Ndeezi G, Mworozi EA, Tumwine JK. Prevalence and factors associated with rotavirus infection among children admitted with acute diarrhea in Uganda. BMC Pediatr. 2010;10:69.

18. Ouyang Y, Ma H, Jin M, Wang X, Wang J, Xu L, et al. Etiology and epidemiology of viral diarrhea in children under the age of five hospitalized in Tianjin, China. Arch Virol. 2012;157(5):881-7.

19. Mackell S. Traveler's diarrhea in the pediatric population: etiology and impact. Clin Infect Dis. 2005;41 Suppl 8:S547-52.

20. Lorrot M, Bon F, El Hajje MJ, Aho S, Wolfer M, Giraudon H, et al. Epidemiology and clinical features of gastroenteritis in hospitalised children: prospective survey during a 2-year period in a Parisian hospital, France. Eur J Clin Microbiol Infect Dis. 2011;30(3):3618.

21. Venkatesh VN, Prashanth HV, Subha DS, Sudhindra KS. Rotaviral Diarrhoea in Children: A Comparison of PAGE with ELISA.J Clin Diagnos Res. 2012;6(2):188-91.

22. Banyai K, Gentsch JR, Martella V, Bogdan A, Havasi V, Kisfali P, et al. Trends in the epidemiology of human G1P[8] rotaviruses: a hungarian study. J Infect Dis. 2009;200 Suppl 1:S222-7.

23. Hassine-Zaafrane M, Sdiri-Loulizi K, Ben Salem I, Kaplon J, Ayouni S, Ambert-Balay K, et al. The molecular epidemiology of circulating rotaviruses: three-year surveillance in the region of Monastir, Tunisia. BMC Infect Dis. 2011;11:266.

24. Madhi SA, Kirsten M, Louw C, Bos P, Aspinall S, Bouckenooghe A et al. Efficacy and immunogenicity of two or three dose rotavirus-vaccine regimen in South African children over two consecu- tive rotavirus-seasons: a randomized, double-blind, placebo-controlled trial. Vaccine. 2012;30 Suppl 1:A44-51.

25. Cunliffe NA, Witte D, Ngwira BM, Todd S, Bostock NJ, Turner AM, et al. Efficacy of human rotavirus vaccine against severe gastroenteritis in Malawian children in the first two years of life: a randomized, double-blind, placebo controlled trial. Vaccine. 2012;30 Suppl 1:A36-43.

26. Farahtaj F, Gallimore CI, Iturriza-Gomara M, Taremi M, Zali MR, Edalatkhah H, et al. Rotavirus VP7, VP4 and VP6 genotypes cocirculating in Tehran, Iran, between 2003 and 2004. Epidemiol Infect. 2007;135(5):834-8.

27. Harrington M, Butler K, Cafferkey M. Rotavirus infection in hospitalised children: incidence and impact on healthcare resources. Ir J Med Sci. 2003;172(1):33-6.

28. Jagai JS, Sarkar R, Castronovo D, Kattula D, McEntee J, Ward H, et al. Seasonality of rotavirus in South Asia: a meta-analysis approach assessing associations with temperature, precipitation, and vegetation index. PLoS One. 2012;7(5).

29. Levy K, Hubbard AE, Eisenberg JN. Seasonality of rotavirus disease in the tropics: a systematic review and meta-analysis. Int $J$ Epidemiol. 2009;38(6):1487-96.

30. Khalili B, Cuevas LE, Reisi N, Dove W, Cunliffe NA, Hart CA. Epidemiology of rotavirus diarrhoea in Iranian children. J Med Virol. 2004;73(2):309-12.

31. Hamedi A, Sadeghian A. Incidence of Rotavirus Diarrhea in Children Under 6 years Referred to the Pediatric Emergency and Clinic of Ghaem Hospital, Mashhad, Iran. Acta Medica Iranica. 2010;48:1-2.

32. Ghorashi Z, Behbahan AG, Oskouei SA. Rotavirus enteric infection in children of northwest Iran. Pediatr Infect Dis J. 2011;30(7):616-8.

33. Amini S, Solati AA, Fayaz A, Mahmoodi M. Rotavirus infection in children with acute diarrhea in Tehran. Med J Islam Repub Iran. 1990;4(1):25-8.

34. Zarnani AH, Modarres S, Jadali F, Sabahi F, Moazzeni SM, Vazirian F. Role of rotaviruses in children with acute diarrhea in Tehran, Iran. J Clin Virol. 2004;29(3):189-93.

35. Samarbafzadeh A, Tehrani EM, Makvandi M, Taremi M. Epidemiological aspects of rotavirus infection in Ahwaz, Iran.J Health Popul Nutr. 2005;23(3):245-9.

36. Momenzadeh A, Modarres S, Faraji A, Sohrabi A, Modarres S, Azarnoush L, et al. Comparison of Enzyme Immunoassay, Immunochromatography, and RNA-Polyacrylamide-Gel Electrophoresis for Diagnosis of Rotavirus Infection in Children with Acute Gastroenteritis. Iran J Med Sci. 2008;33(3):173-6.

37. Nagaoka Y, Tatsumi M, Tsugawa T, Yoto Y, Tsutsumi H. Phylogenetic and computational structural analysis of VP7 gene of group a human rotavirus G1P[8] strains obtained in Sapporo, Japan from 1987 to 2000. J Med Virol. 2012;84(5):832-8.

38. Arora R, Chitambar SD. Full genomic analysis of Indian G1P[8] rotavirus strains. Infect Genet Evol. 2011;11(2):504-11.

39. Cashman O, Collins PJ, Lennon G, Cryan B, Martella V, Fanning S et al. Molecular characterization of group A rotaviruses detected in children with gastroenteritis in Ireland in 2006-2009. Epidemiol Infect. 2012;140(2):247-59. 